\title{
Paciente con amputación transfemoral derecha, clavo gamma y síndrome hemimotor derecho: valoración y manejo del proceso de protetización
}

\author{
Patient with a right transfemoral amputation, gamma nail, and right Hemi-motor syndrome: \\ assessment and management of the prosthetic fitting
}

\author{
Rossana Chiesa Estomba ' , Concepción Cuenca González ${ }^{1}$, Miriam Robledo García ' , Arsenio Granja Requena 1 , \\ Luis Monleón Llorente L'y Lucia Garvín Ocampos L'
}

\section{Resumen}

Introducción: la decisión de protetizar a un paciente amputado depende de: la etiología, nivel de amputación, uni o bilateralidad, comorbilidades, soporte sociofamiliar, capacidad funcional y motivación del paciente. Observación clínica: varón, 83 años, diabético, cardiopatía isquémica, accidente cerebrovascular con paresia braquial derecha residual en año 2000. Clavo gamma por fractura pertrocantérea derecha en 2012 y amputación transfemoral derecha en 2018 de causa vascular. Se protetiza mediante encaje de contención isquiática CAT-CAM, sistema de suspensión tipo Kiss, rodilla de bloqueo a la extensión con adaptación del sistema de desbloqueo en porción antero-interna del encaje y pie Sach. Tras tratamiento fisioterápico, consigue deambulación autónoma con ayuda de un bastón y tercera persona por dificultad para agarre de MSD. Conclusión: los médicos rehabilitadores debemos apostar por dotar a nuestros pacientes de autonomía y funcionalidad, siempre que sea viable.

Palabras clave: amputación transfemoral; protetización; accidente cerebrovascular

\begin{abstract}
Introduction: prosthesis, an amputee patient, depends on etiology, level, uni or bilaterality, comorbidities, socio-family support, functional capacity, and motivation of the patient. Clinical observation: male, 83 years old, diabetic, ischemic heart disease, stroke with residual upper right limb (URL) paresis in 2000. Gamma nail due to the right trochanteric fracture in 2012, and right transfemoral amputation in 2018 of vascular cause. Prosthetic fitting CAD-CAM socket/interface, Kiss type suspension system, extension locking knee with an adaptation of the unlocking system in the antero-internal portion of the socket, and Sach foot. After physiotherapeutic treatment, he achieves independent walking with the help of 1 cane and third-person due to difficulty in the URL grip. Conclusion: rehabilitation doctors must bet on providing our patients with independency, and better function, whenever it is viable.
\end{abstract}

Keywords: transfemoral amputation; protection; stroke

Fecha de envío: 6 de noviembre de 2019 - Fecha de aceptación: 23 de junio de 2020

\section{Introducción}

En Estados Unidos en el año 2005, la prevalencia de pacientes con pérdida de miembros inferiores era de 1,6 millones. Se estima que para 2050 esta cifra se elevará a 3,6 millones de personas. La mayoría de los casos será por etiología vascular como la enfermedad arterial periférica y diabetes mellitus (DM), comúnmente asociada a amputaciones vasculares. Las amputaciones son más frecuentes en pacientes mayores, de sexo masculino y raza negra (aumento de 27\% de 1988 a 1997 por cada 100000 nacidos vivos). Las de origen traumático y oncológico $(-50 \%$ y $-43 \%$ por cada 100000 nacidos vivos de 1988 a 1997, respectivamente), así como las agenesias, están en declive (Varma et al., 2014).

(1) Servicio de Medicina Física y Rehabilitación, Hospital Clínico San Carlos, Madrid, España.

Autor de correspondencia: chiesarossap@gmail.com 
La incidencia de patología vascular de miembros inferiores aumenta con la edad, así como con las comorbilidades como la diabetes mellitus. En Inglaterra más del $90 \%$ de las amputaciones son realizadas en pacientes mayores de 50 años y son secundarias a enfermedad vascular (Stevens et al., 2018).

Según sea la afectación que presente el miembro inferior se decidirá, de forma consensuada, el cirujano vascular-cirujano ortopédico y el médico rehabilitador cual es el mejor nivel de amputación que se realizará al paciente, que permita la mayor funcionalidad posible. Los niveles de amputación se clasifican en menores, aquellos restringidos al pie (Chopart, Lisfranc, transmetatarsiana, dedos), y mayores, por encima del tobillo (Syme) hasta la cadera (hemipelvectomia). El nivel de amputación más frecuente, de forma global, es el del primer dedo del pie. Entre los niveles de amputación mayor de miembros inferiores, el más frecuente es el transtibial, seguido por el transfemoral (Cifu, 2016). Las tasas de supervivencia tras amputación varían según la etiología, siendo esta menor en amputaciones de causa vascular y variando al mes, al año y a los 5 años, donde puede superar el $50 \%$. La protetización no parece estar asociada con la reducción del riesgo de presentar un evento cardíaco importante después de la amputación (Chamlian et al., 2014; Cifu, 2016; Mundell et al., 2018).

El 97\% de las amputaciones de origen vascular son las de miembro inferior. La DM representa el $67 \%$ de todas las amputaciones. Al ajustar la tasa de amputación por edad, se ha encontrado que es entre 18 y 28 veces mayor en diabéticos que en personas no diabéticas. Por otra parte, el hábito tabáquico se ha asociado a un aumento en el riesgo de re-amputación hasta 25 veces mayor con respecto a los no fumadores (Cifu, 2016). En los pacientes en los que la amputación inicial ha sido de causa isquémica, se ha descrito un riesgo de re-amputación 4 veces mayor que en pacientes con úlceras cutáneas (Torbjörnsson et al., 2020). En el análisis publicado en 2017, Vascular Quality Initiative (VQI) realizado entre 2013 y 2015, determina que la ratio o proporción de amputaciones por debajo de la rodilla (BKA) y por encima de la rodilla (AKA) fue de $1,29: 1$, con un $43 \%$ de ellas con historia previa de revascularización de la extremidad. El porcentaje de re-intervención fue mayor en amputaciones trastibiales, 5\% de los casos, en comparación con el $1 \%$ de amputaciones por encima de la rodilla. Un tercio de los pacientes con BKA eran menores de 60 años, tenían mayor prevalencia de diabetes mellitus y una amputación menor previa de esa extremidad, a diferencia de la población de mayor edad, de causa isquémica con AKA inicial (Gabel et al., 2017).

Comentamos el caso clínico sobre la decisión de protetización de un paciente de nuestro Servicio de Rehabilitación con amputación transfemoral secundaria a enfermedad isquémica arterial y secuelas de accidente cerebro vascular (ACV) de arteria cerebral media izquierda.

\section{Caso clínico}

Varón de 83 años, con antecedentes personales de DM, Hipertensión arterial (HTA), cardiopatía isquémica y ACV en arteria cerebral media izquierda con hemiplejia derecha de predominio braquial residual desde el año 2000. Es portador de una válvula protésica aórtica metálica en 2006 y de clavo gamma por fractura pertrocantérea derecha en 2012.

Su situación basal funcional era de independencia para las actividades básicas de vida diaria, excepto baño y aseo personal con ayuda de tercera persona, marcha independiente por interiores y supervisada por exteriores sin ayudas técnicas, recorriendo distancias superiores a 500 metros en terreno llano, siendo categorizado con un nivel de actividad K2.

El paciente presenta una amputación transfemoral derecha, de causa vascular, realizada en 2018 y con los antecedentes previos descritos se plantean las alternativas terapeúticas de realizar protetización o solamente enseñar medidas higiénico-posturales del muñón, aprendizaje de transferencias y manejo de silla de ruedas manual para desplazamientos tipo Arnás de palanca que permite dirigir la silla de ruedas con una mano (especialmente indicada para pacientes con paresias).

Debido al buen soporte familiar, disposición favorable del paciente y consecución de un adecuado reaconcionamiento físico, consiguiendo apoyo monopodal estable en paralelas y con andador, se inicia protetización con prescripción de encaje de contención isquiática CAT-CAM, sistema de suspensión tipo Kiss, especialmente sencillo para uso en pacientes geriátricos y pacientes que presentan restricción de la motricidad fina de manos, rodilla de bloqueo manual a la extensión, con adaptación del sistema de desbloqueo en parte anterior-interna del encaje ( habitualmente se aloja en borde superoexterno del encaje) (Figura 1) que le facilita un acceso más cercano a la mano izquierda, única funcional del paciente y un pie con talón almohadillado SACH (Solid Ankle Cushion Heel) de bajo peso, por su bajo coste económico y ligereza, hasta estar seguros de conseguir el objetivo de marcha segura con la prótesis, dadas las limitaciones previas con las que nos enfrentamos en este caso de protetización, valorando la posibilidad de cambio de pie en un futuro por un pie articulado. Nuestro objetivo es conseguir una marcha segura, lo más independiente posible, con el menor consumo energético por sus factores de riesgo cardiovascular presentes y que impida caídas u otras complicaciones en nuestro 
paciente, siendo conscientes de que, para este objetivo, no hemos utilizado inicialmente el pie más adecuado. La rodilla prescrita dispone de un sistema hidráulico que le permite realizar una sedestación controlada al realizar una flexión lenta de la rodilla de la prótesis (3R31 Prosedo ${ }^{\circledR}$ rodilla Ottobock) (Figura 2).

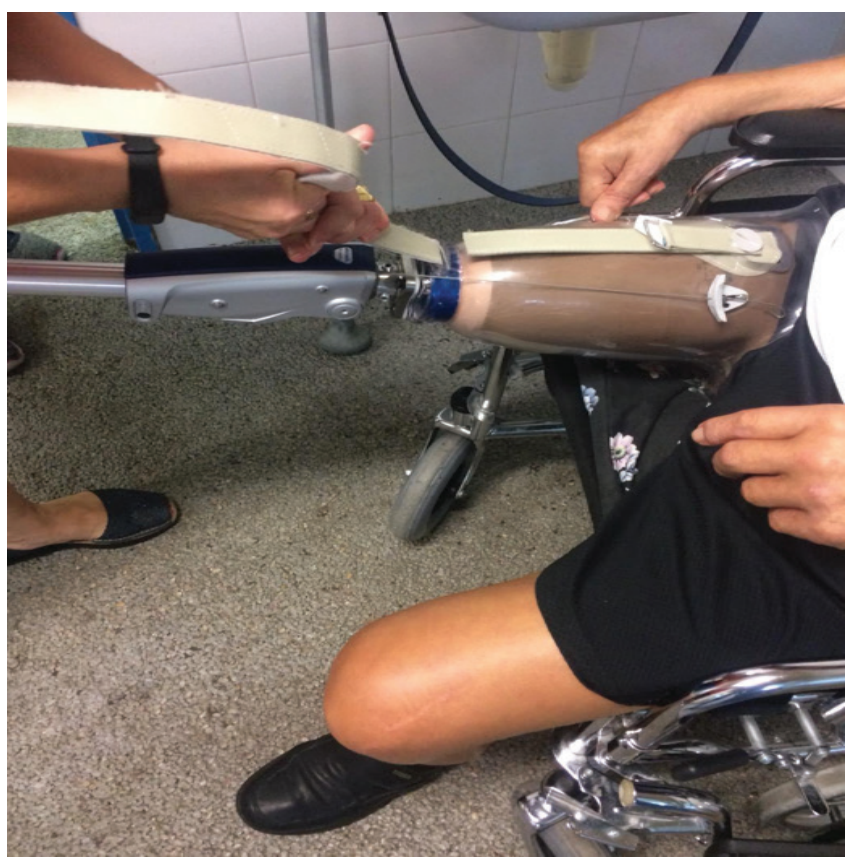

Figura 1: Encaje de contención isquiática CAT-CAM, sistema de suspensión tipo Kiss. Rodilla de bloqueo en extensión

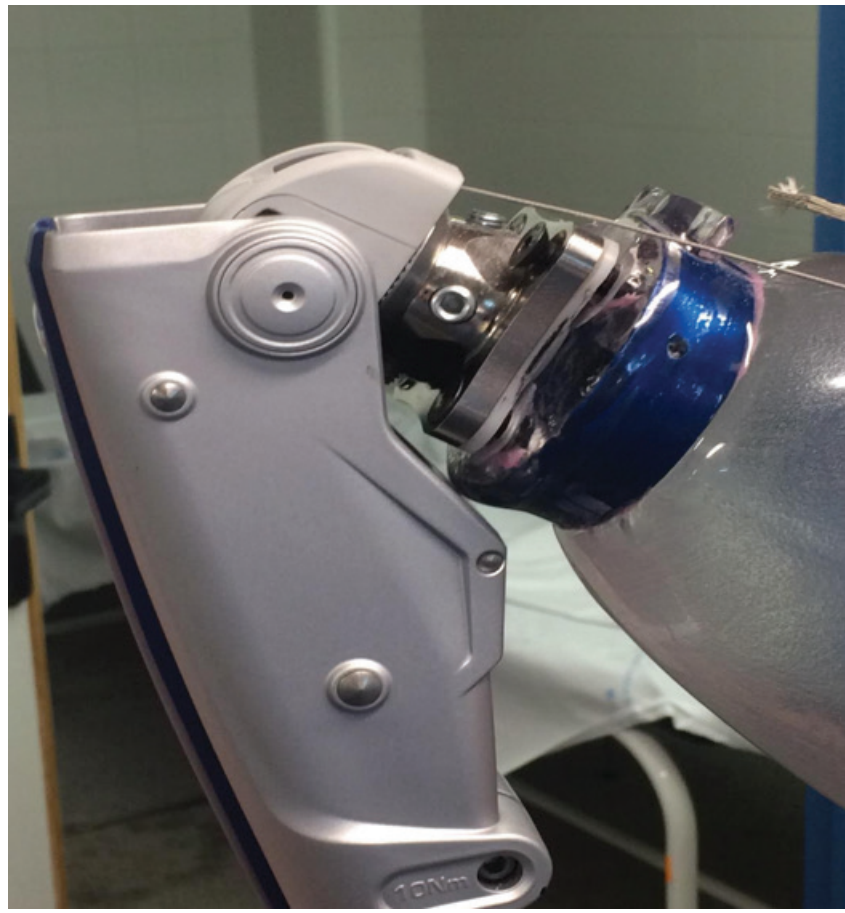

Figura 2: Rodilla con sistema hidráulico (3R31 prosedo ${ }^{\circledR}$ rodilla Ottobock)
En la exploración física observamos un muñón de amputación bien conformado, sin complicaciones cutáneas, no doloroso a la palpación y sin evidencia de neuromas ni esquirlas óseas, de $14 \mathrm{~cm}$ de longitud medidos desde trocánter menor. La fuerza muscular (FM) del hemicuerpo izquierdo es de 5/5 (deltoides, bíceps, tríceps, extensores del carpo, flexores de dedos, psoas, cuádriceps, isquiotibiales, tibial anterior, tríceps sural y extensor del primer dedo del pie) según la escala de Daniels, y la del miembro superior derecho (MSD) es de 0/5, pléjico (deltoides, bíceps, tríceps, extensores del carpo, flexores de dedos). El muñón presenta una FM de 4/5 (psoas, cuádriceps, adductores, glúteo mayor y medio), con un balance articular sin limitación a la extensión completa del muñón, prueba de Thomas y Ober negativas. El pie del miembro inferior izquierdo no presentaba cambios de coloración ni úlceras vasculares.

Para conformar el muñón se realizan autovendajes diurnos y nocturnos con venda de baja elasticidad (comprilam ${ }^{\circledR}$ ) que favorece la reducción volumétrica y del edema del mismo, consiguiéndose dicho objetivo en 4 semanas tras la amputación. Tras estabilización circométrica inicial, comenzamos con la adaptación de liner de silicona.

Para reducir el gasto económico al paciente, en el proceso de protetización, utilizamos este vendaje de baja elasticidad que nos permite no prescribir un socket de reducción inicial de alto coste económico para el escaso tiempo de uso, pasando a utilizar el liner definitivo, una vez conseguida la estabilización circometría en una semana, tomadas de forma secuencial, de 5 en 5 centímetros, desde periné hasta el extremo distal del muñón.

Se instruye al paciente en el cuidado de la piel del muñón realizando hidratación nocturna, con la aplicación de crema de pH neutro, y secado matutino de la piel con alcohol de romero previo a la colocación del liner, para evitar la posible maceración de la piel del muñón provocada por la falta de transpiración de la silicona.

El paciente consigue realizar apoyo monopodal en paralelas, con fuerza muscular de 5/5 en hemicuerpo izquierdo y muñón aceptable (fuerza muscular psoas 4/5, glúteo medio 4/5, cuadrado lumbar $5 / 5$ y aductores $4 / 5)$.

Durante el proceso de protetización, 6 semanas desde la amputación hasta la entrega de la prótesis con encaje de chequeo provisional, se revisó el muñón del paciente en busca de zonas de hiperpresión o roce que pudieran producir complicaciones cutáneas como erosiones, úlceras, ampollas o hematomas. 
Se prescribe, como interfase con el encaje rígido, un liner de silicona, que debe retirar durante la noche. Las modificaciones planteadas en el encaje y la alineación de la prótesis son realizadas, bajo la supervisión del médico rehabilitador, por el técnico protésico (integrante del equipo multidisciplinar). Él será el encargado de elaborar un encaje provisional inicial a medida previo toma de molde por sistema CAT-CAM. El encaje definitivo, con las modificaciones realizadas para adaptarlo a los cambios volumétricos del muñón, será de tipo laminado.

Estas modificaciones iniciales consisten en el ajuste de la altura de la rama isquiática, para evitar roces cutáneos en dicha zona y en región del periné, relleno del encaje mediante foam, para evitar presencia de inestabilidad mediolateral del encaje-munón por la disminución del volumétrica que siempre se produce al comenzar a realizar carga del muñón con la prótesis y ajuste de la altura del tubo endoesqueletico de la prótesis para evitar la aparición de un mal patrón postural y de alteraciones de la marcha con posibles sobrecargas en la región lumbar. La alineación de la prótesis se realizó en taller ortopédico externo al hospital, haciendo, inicialmente, la alineación de banco para el ensamblaje correcto de los componentes de la prótesis; la alineación estática se realiza con la prótesis adaptada al muñón mediante alineador con laser LASAR (Lasser Assisted Static Alignment Reference). Posture para comprobar que la posición del encaje con respecto al eje de la rodilla, pierna y pie es igual que a la alineación de banco.

El encaje se ha alineado con $5^{\circ}$ de flexión en el plano sagital y $5^{\circ}$ de aducción en el plano frontal (por la abducción fisiológica del muñón). En el plano frontal, hicimos coincidir el centro de la rodilla con el centro del tobillo y la vertical, que une ambas articulaciones, hacerla pasar entre la mitad y el tercio interno de la pared posterior de encaje. En el plano sagital esta vertical se continua en un punto situado en la mitad del borde interno del encaje, dejando el eje de carga ligeramente retrasado para dar mayor estabilidad en la prótesis y mejorar el equilibrio del paciente, favorecido por la rodilla de bloqueo que tiene adaptada su prótesis.

Por último, la alineación dinámica se hizo de forma visual y mediante videocámara utilizada en el pasillo de nuestro servicio y con espejo en gimnasio, por no disponer de sistema de análisis instrumentado en un nuestro hospital. Los talones de los pies (protésico y natural) los dejamos separados a $10 \mathrm{~cm}$, con ligera rotación externa de $10^{\circ}$, observando que el pie protésico tiene suficiente equino para contactar suavemente con el suelo sin que exista roce de la punta con el suelo
Se realiza regulación de la altura de la prótesis, dejándola $1 \mathrm{~cm}$ más baja de la altura del miembro sano para favorecer la progresión de la prótesis durante la marcha, evitando levantar excesivamente la cadera del lado amputado o rozar la punta del pie prótesico con riesgo de caida. Tras conseguir una adecuada tolerancia del encaje de chequeo, se realiza laminado definitivo.

Se le prescribe tratamiento de fisioterapia durante 6 meses, consistente en cinesiterapia (CNT) analítica del muñón, fortalecimiento de hemicuerpo izquierdo, reeducación postural para compensación del lado pléjico, propiocepción y equilibrio y reeducación de la marcha, primero en paralelas, después con andador y finalmente con muleta en lado sano y 1 persona en miembro superior pléjico (Figura 3).

Consiguió subir y bajar escaleras, realizar deambulación en rampa y tener autonomía para la colocación de la prótesis con su mano funcional izquierda. El paciente es capaz, al finalizar el proceso de protetización, de realizar deambulación con ayuda de 1 bastón y de $3^{\circ}$ persona por incapacidad para el agarre con su mano derecha, planteándonos la posibilidad de uso de bastón de apoyo axilar en miembro superior derecho.
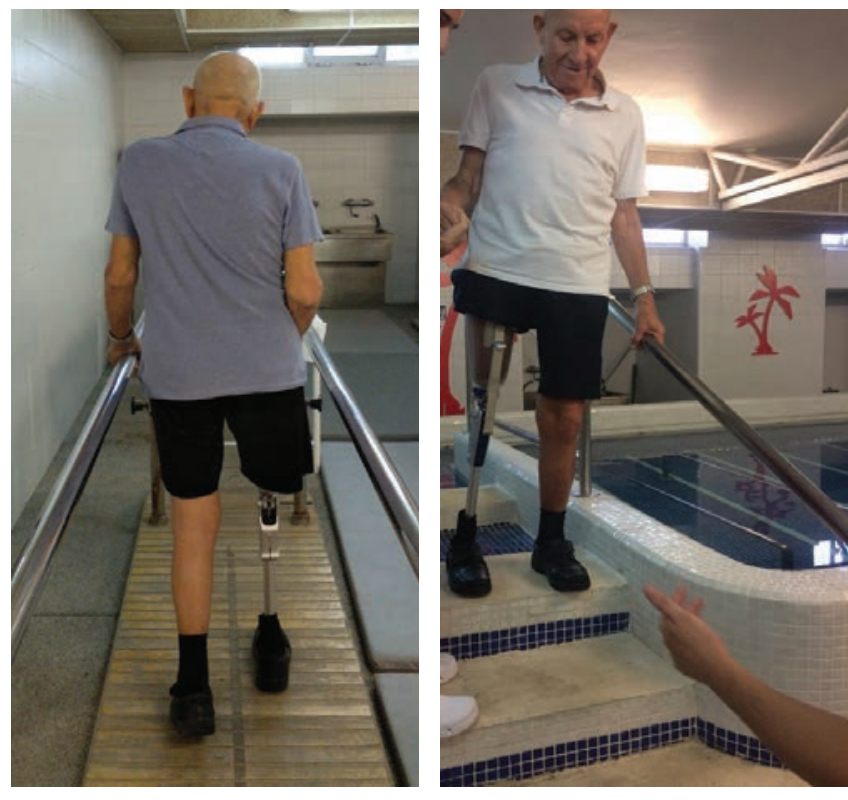

Figura 3: Apoyo estable es paralelas y escaleras con prótesis transfemoral

\section{Discusión}

La enfermedad arterial periférica continúa siendo la principal causa de amputación de miembro inferior a nivel mundial siendo la prevalencia mayor en el sexo masculino, por encima de los 60 años de edad. La edad del paciente podría dificultar la protetización, 
aunque, como se ha visto en otros estudios, no debe considerarse de forma aislada como contraindicación absoluta para la protetización, pudiendo influir en la consecución de la marcha (Chamlian et al., 2014).

La presencia de un mayor número de comorbilidades como enfermedad renal crónica avanzada, ictus previo, deterioro cognitivo, entre otros; la mala condición física previa, edad avanzada, y el tipo de amputación (amputaciones altas), condicionan de forma negativa las posibilidades de recuperación funcional tras la amputación de miembros inferiores (Chamlian et al., 2014; Meier et al., 2014; Saeed, 2015; Cifu, 2016; Moreno- López et al., 2017 ). El conocimiento de todos estos factores desfavorables para la consecución de una protetización exitosa fueron los que nos hicieron plantearnos inicialmente la no protetización del paciente. Por otro lado, al presentar plejia del miembro superior derecho sabíamos que esto nos dificultaría un adecuado patrón de marcha por la ausencia de braceo y la tendencia a la carga en el hemicuerpo izquierdo.

La amputación debe ser llevada a cabo por un cirujano experto, habitualmente cirujano vascular para los procesos de causa isquémica y cirujano ortopédico para los de causa traumática y oncológica, con una selección adecuada del nivel de amputación en cada caso.

En el postoperatorio, se procede al vendaje de conformación del muñón, con las correspondientes curas periódicas por el cirujano durante algo más de dos semanas (Klarich et al., 2014). El muñón femoral debe medir al menos $10-15 \mathrm{~cm}$ desde el trocánter menor para conseguir una correcta protetización. Para la colocación de rodilla protésica es preciso disponer de 10 a $12 \mathrm{~cm}$ de distancia desde el extremo del muñón hasta la línea intercondílea de rodilla, ya que esto condiciona la necesidad de adaptar una rodilla policéntrica de 4 barras cuando no se cumplen estas condiciones (en muñones largos el espacio para alojar la rodilla protésica es menor). La cicatriz postquirúrgica no debe estar adherida, debe ser móvil y tener una buena almohadilla de tejidos blandos, sin signos de infección, secreciones, eritema o hemorragia, reducción de edema, volumen del muñón adecuado y ausencia de lesiones cutáneas con adecuada sensibilidad. También la ausencia de dolor a la palpación en la cicatriz favorece la buena evolución, así como la estabilidad del muñón con fuerza muscular de agonista y antagonista adecuada y el biselado correcto óseo distal (Zambudio, 2009; Mendoza- Cruz et al., 2014; Cifu et al., 2016; Rodeiro, 2018).

El proceso de amputación consta de una fase prequirúrgica, en la que se valora la situación clínica del paciente y se le informa acerca del proceso; una fase posquirúrgica inmediata, en la que el médico rehabilitador inicia los cuidados del muñón y la higiene postural del mismo; una fase preprotésica en la que se continúan los cuidados del muñón, se mantienen rangos articulares proximales al muñón ( $0^{\circ}$ extensión, $120^{\circ}$ flexión) y se inician ejercicios de potenciación muscular, en el caso de nivel transfemoral los músculos glúteo mayor (que evitará flexo de cadera y sustituye a cuádriceps), glúteo medio (estabilización lateral, impide claudicación de lado protésico), aductores (impide actitud en ABD de muñón), cuadrado lumbar (agonista de despegue y elevación de la prótesis), también pelvitrocantéreos, abdominales y paravertebrales (Rodeiro, 2018), trabajando el apoyo unipodal sobre el miembro sano, el equilibrio $y$, finalmente una fase protésica, en la que se prescribe el tipo de prótesis más adecuado para el paciente y la adaptación de la misma mediante un encaje provisional inicial y uno laminado al finalizar el proceso. El chequeo es realizado por el médico rehabilitador, en colaboración con el técnico ortopédico que realizará las modificaciones indicadas. En la fase final, se incorporará el entrenamiento en las actividades de la vida diaria, la reeducación de la marcha y el aprendizaje del modo de retirar y colocar la prótesis, entre otros (Cifu, 2016; Mendoza- López et al., 2017).

Los componentes a utilizar en una prótesis transfemoral son: encaje, rodilla, pie y sistema de suspensión. Pueden adaptarse desrrotadores y sistemas de amortiguación. Actualmente se prescriben con más frecuencia las prótesis endoesqueléticas, con el encaje exterior duro, interfase blanda y tubo de soporte interior, con media y funda estética exterior. Las prótesis endoesqueléticas con tubo de soporte de carga interior, son más ligeras, permite alineación más rápida sin desmontar componentes. La prótesis exoesquelética posee una capa exterior dura de soporte de carga, es económica, duradera y protege la prótesis en ambientes hostiles, aunque la principal desventaja es el peso añadido, las opciones limitadas de pie y rodilla, la reparación de componentes y alineación limitada ( Zambudio 2013; Miller 2015; Cifu 2016), quedando su prescripción relegada, en nuestro entorno asistencial, para el uso en el baño, en mar y piscina, disponiendo un agujero o sistema de llenado (para que no flote) y de drenaje para salir el agua.

Algunos encajes, como el triangular, está en desuso. Lo mismo ocurre con el encaje cuadrangular que se caracteriza por mayor diámetro mediolateral que anteroposterior. El encaje de contención isquiática es el más usado y se caracteriza por su forma rectangular, con un lado mayor anteroposterior y más estrecho en sentido mediolateral que permite una mayor tolerancia a nivel de isquion y glúteos, con mejor tolerancia y menor consumo energético durante la marcha. Otros tipos de encajes son el ISNY y MAS (Zambudio, 2009; Miller, 2015; Cifu, 2016). Es importante, junto con el encaje, decidir el sistema de suspensión a utilizar que mantendrá la prótesis 
anclada al muñón, pudiendo ser de succión (con tapón o válvula que cierra orificio en parte distal del encaje, creando una presión negativa que se sella con la válvula al introducir el muñón), cinturón de suspensión elástica, cinturón pélvico tipo socket, sistema de PIN o perno, y sistema de válvula de expulsión (que puede llevar tubo conectado al pie). (Kelly et al., 2007; Cifu 2016).

El tipo de articulación de rodilla a utilizar es determinante ya que contribuye a conseguir un apoyo seguro y un adecuado movimiento de flexo-extensión durante la marcha. También debe permitir al paciente sentarse, flexionar las piernas o arrodillarse, siempre teniendo en cuenta su seguridad. Pueden ser de un eje (monocéntricas) o de varios ejes (policéntricas). Deben acompañarse de un sistema de estabilización y de control de movimiento. La rodilla con bloqueo manual posee un mecanismo automático de bloqueo que se activa con la extensión del muñón y se desactiva de forma manual, pudiendo caminar con la rodilla bloqueada en extensión y desbloquearla manualmente para sentarse. El patrón de marcha no es el mejor, pero ofrece la mayor estabilidad disponible y estaría indicada en pacientes geriátricos, menos acondicionados o con comorbilidades y con mayor inestabilidad de la marcha, como es nuestro caso (Kelly et al., 2007; Zambudio, 2009; Miller, 2015). Otros tipos de rodilla son: rodilla de un solo eje (asiste en extensión para avance rápido del pie en balanceo, precisa de fuerza proximal para evitar inestabilidad), de control por peso o freno a la carga (mecanismo de bloqueo que se activa por peso en apoyo, flexión en descarga), policéntricas (eje múltiple, ideal en desarticulación de rodilla por menor distancia entre eje de rodilla y unión del encaje), hidráulicas (pistones con fluido) y neumáticas (pistones con aire); estás dos últimas permiten mayor control en balanceo y se ajustan a cambios en la velocidad de la marcha. Por último, las rodillas con microprocesadores, que utilizan sensores para detectar el movimiento, el ritmo y se ajustan al tipo de marcha (Zambudio 2009; Cifu, 2016).

Existe una variedad importante de pies protésicos. Deben proporcionar una superficie estable para la carga, absorber la energía del impacto, reemplazar la función de la musculatura perdida, reproducir la biomecánica del pie y tobillo y también ser estéticamente adecuados. Pueden ser dinámicos (con retorno de energía) o estáticos (sin retorno de energía); no articulados como el pie con talón almohadillado (SACH) ideal para pacientes con baja demanda, y el pie SAFE (Solid ankle flexible endoskeleton); articulados, como el pie de eje único, de eje multiaxial, e hidraúlico; pies con retorno de energía y de respuesta dinámica con perfil bajo y alto y, por último, los más avanzados, que son pies con sistema de control por microprocesador para actividad especial (Kelly et al., 2007; Zambudio, 2009; Miller, 2015; Cifu, 2016).
Nuestro paciente presentaba comorbilidades importantes, edad avanzada y amputación mayor de nivel alto (transfemoral), con monoparesia derecha como secuela de ictus, lo cual nos supuso un reto importante para protetizar.

Observando la buena evolución clínica tras comenzar la cinesiterapia, consiguiéndose un adecuado re-acondionamiento físico, el importante soporte familiar que tenía, fuerte motivación y buen ánimo se decide finalmente protetizar.

La alineación correcta de la prótesis es importante para conseguir la funcionalidad y comodidad adecuada. Se define como la relación entre el encaje y el resto de componentes de la prótesis. Para que la alineación sea óptima debe realizarse de forma estática, dinámica y la alineación de banco (Lusardi et al., 2013). La alineación de banco corresponde al ensamblaje de los componentes de la prótesis como lo especifica el fabricante, garantiza la estabilidad de la rodilla alineando el eje protésico. La línea de carga debe pasar por el centro de la rodilla y el tobillo en el plano frontal, y por el tercio interno del encaje. Si es preciso conseguir más estabilidad se retrasará el eje (Zambudio, 2009). Con el individuo en la postura LASAR (laser-assisted static alignment reference) se evalúa la alineación de la prótesis en estático, se observa si la prótesis es capaz de soportar la trasmisión del peso, la correcta nivelación de la pelvis, estabilidad de la rodilla y distancia de la base de apoyo (Lusardi et al., 2013). Finalmente se pide al paciente que camine con la prótesis y se valora la alineación dinámica, observando la armonía de la marcha y el gasto energético (Zambudio, 2009).

Según se recoge en los estudios el gasto energético medido por el consumo de $\mathrm{O} 2$ (VO2) para pacientes con amputación transfemoral de causa no vascular es $30 \%$ a $60 \%$ mayor si se compara con la población de misma edad y sexo no amputada, y se incrementa a medida que aumenta la velocidad de marcha. Los amputados transfemorales de causa no vascular, presentan un menor consumo de oxígeno a mayor Self-Selected Walking Speed (SSWS) con respecto a amputados transfemorales de causa vascular, quienes incluso a una velocidad de marcha menor presentan una carga metabólica mayor. Se utiliza el SSWS o velocidad de marcha confortable, para calcular el coste de energía relativa en pacientes con discapacidad. El SSWS en pacientes amputados femorales es más lento con respecto a los no amputados y amputados de causa no vascular, incluso a velocidad de $0,6 \mathrm{~m} / \mathrm{s}$ los amputados de causa vascular están al $60 \%$ de su capacidad aeróbica máxima. Esto tiene relación con el esfuerzo percibido y es probable que los amputados elijan caminar en un SSWS más lento, aunque resulte menos económico para evitar una carga metabólica (Relative Metabolic Load, RML) mayor. Algunos autores sugieren que el entrenamiento aeróbico puede 
reducir clínicamente la RML en amputados de causa vascular, así como contribuir a una marcha más económica (Wezenberg et al., 2013). En el caso de las amputaciones de causa vascular, algunos estudios sugieren un mayor gasto energético, que puede variar según los componentes de la prótesis. Isakov et al. Compararon la frecuencia cardíaca, velocidad de marcha y consumo de oxígeno en amputados transfemorales de causa vascular versus amputados transfemorales de causa traumática con rodilla de mecanismo de bloqueo y rodilla sin él, en ambos grupos. Obtienen que el uso de rodilla con bloqueo generó un aumento menor de frecuencia cardíaca respecto a sistema abierto; el SSWS fue mayor con la rodilla de bloqueo en pacientes de mayor edad, el consumo de oxígeno fue mayor con rodilla sin bloqueo; por todo esto recomiendan en pacientes amputados mayores el uso de rodilla con bloqueo ya que permite mayor velocidad de marcha con menor esfuerzo. (Isakov et al., 1985; Cznerniecki et al., 2015; Mengelkoch et al., 2017; Van Schaik et al., 2019)

Las predicciones del modelo en este estudio indican que mejorar el pico de VO2 a través del entrenamiento aeróbico puede resultar en una reducción clínicamente relevante del VO2rel en personas con amputación vascular. Además, puede permitir a las personas adoptar una velocidad de caminata más rápida y económica. Por lo tanto, proponemos que la capacidad aeróbica máxima se considere como un factor importante cuando se busca mejorar la capacidad de caminar en personas con una amputación.

Se necesitan estudios longitudinales para evaluar empíricamente el efecto predicho del entrenamiento aeróbico sobre la capacidad de caminar en adultos mayores con una amputación de miembros inferiores.

El objetivo de la protetización es conseguir que el paciente sea lo más funcional posible. En la fase preoperatoria, se pueden realizar ejercicios de reacondicionamiento, equilibrio, fortalecimiento de la musculatura de tronco y MMSS. Tras la amputación, comienzan con el entrenamiento de transferencias y posicionamiento del miembro afectado, fundamental para evitar las contracturas. Progresivamente, se debe iniciar marcha en paralelas y con bastones, así como potenciación muscular. Deben adaptarse bastones y silla de ruedas para los traslados, con el objetivo de disminuir el riesgo de caídas (Zambudio, 2009; Klarich et al., 2014).

\section{Conclusión}

Los médicos rehabilitadores debemos apostar por dotar a nuestros pacientes de la máxima autonomía y capacidad funcional posible, siempre que esta sea viable y contemos con suficiente motivación por parte del paciente y soporte familiar adecuado.
Es importante disponer de un equipo multidisciplinar formado por profesionales especializados (médico rehabilitador, fisioterapeutas, técnicos ortoprotésicos, cirujanos ortopédicos-vasculares, terapeutas ocupacionales, psicólogos y trabajadores sociales) para poder llevar a cabo una satisfactorio proceso de protetización de nuestros pacientes, informándoles en cada momento de la evolución de su proceso, de las posibles complicaciones que puedan generarse, pactando objetivos realistas y consensuando las posibles alternativas terapéuticas disponibles, obteniendo los mejores resultados funcionales y emocionales, con las mínimas dificultades y la máxima adherencia para el uso de su prótesis.

\section{Agradecimientos}

Agradecemos la colaboración del paciente y su familia para poder realizar este trabajo.

\section{Fuentes de financiamiento}

El equipo investigador declara no tener ninguna fuente de financiamiento para la realización de este trabajo.

\section{Contribuciones y conflictos declarados por los autores}

El equipo investigador declara no tener ningún conflicto de interés. Cada autor ha contribuido al tratamiento y seguimiento del paciente, así como con la redacción y revisión de este artículo.

\section{Referencias}

Chamlian TR. (2014) Use of prostheses in lower limb amputee patients due to peripheral arterial disease. Einstein (Sao Paulo) 12, 440-446.

Cifu DX. (2015) Braddom's physical medicine \& rehabilitation. Elsevier.

Czerniecki JM \& Morgenroth DC. (2017). Metabolic energy expenditure of ambulation in lower extremity amputees: what have we learned and what are the next steps? Disabil Rehabil. 39, 143-51.

Eva Et T, Fagerdahl A-Mf AM, Blomgren LBI L, Boström Lb L, Ottosson Co C \& Malmstedt Jm J. (2020). Risk factors for re-amputations in patients amputated after revascularization for critical limb ischemia. J Vasc 5214, 31084-3.

Gabel J et al. (2018). Analysis of Patients Undergoing Major Lower Extremity Amputation in the Vascular Quality Initiative. Annals of vascular surgery $46,75-82$.

Isakov E, Susak Z \& Becker E. (1985). Energy expenditure and cardiac response in above-knee amputees while using prostheses with open and locked knee mechanisms. Scand J Rehabil Med Suppl. 12, 108-111. 
Chiesa et al.

Kelly BM, Spires MC \& Restrepo, JA. (2007). Orthotic and prosthetic prescriptions for today and tomorrow. Physical medicine and rehabilitation clinics of North America 18, 785-858.

Klarich, J. \& Brueckner I. (2014). Amputee rehabilitation and preprosthetic care. Physical medicine and rehabilitation clinics of North America 25, 75-91.

Lusardi MM, Jorge M. \& Nielsen C. (2013) Orthotics and Prosthetics in Rehabilitation. In: Orthotics and Prosthetics in Rehabilitation. Elsevier H. 24, 652-84.

Mark D \&Miller J HA. (2009) Rehabilitación: marcha, amputaciones, prótesis, ortesis y lesiones neurológicas. In: España. E, editor. Ortopedia y Traumatología: Revisión Sistemática. Fith. Elsevier;. p. 560-78.

Mengelkoch LJ, Kahle JT \& Highsmith MJ. (2017) Energy costs and performance of transfemoral amputees and non-amputees during walking and running: A pilot study. Prosthet Orthot Int. 41, 484-91.

Periago RZ. (2013)Prótesis, ortesis y ayudas técnicas. Journal of Chemical Information and Modeling. 1689-1699
Rodeiro MG. (2018) Guía de atención de pacientes amputados. Ministerio de Salud. Secretaría de Políticas Regulación e Instutos.;. p. 1-15. Accedido en https://www.argentina.gob.ar/inareps/ guia-atencion-pacientes-amputados el 20 de marzo de 2020

Saeed AB, Saeed UB, Zain-Ur-Rehman M, Ahmad Khan RD \& Yasin, A. (2015). Factors affecting functional outcome after lower extremity amputation. JPMA. The Journal of the Pakistan Medical Association 65, S220-S224.

Stevens PM, Rheinstein J. \& Wurdeman SR. (2018). Prosthetic Foot Selection for Individuals with Lower-Limb Amputation: A Clinical Practice Guideline. Journal of prosthetics and orthotics:JPO 30, 175-180.

Van Schaik L, Geertzen J, Dijkstra PU. \& Dekker R. (2019). Metabolic costs of activities of daily living in persons with a lower limb amputation: A systematic review and meta-analysis. PloS one 14, e0213256.

Varma P, Stineman MG. \& Dillingham TR. (2014). Epidemiology of limb loss. Physical medicine and rehabilitation clinics of North America 25, 1-8.

Wezenberg D, van der Woude LH, Faber WX, de Haan A. \& Houdijk H. (2013). Relation between aerobic capacity and walking ability in older adults with a lower-limb amputation. Archives of physical medicine and rehabilitation 94, 1714-1720. 